\title{
Multi-Symplectic Magnetohydrodynamics: II, Addendum and Erratum
}

\author{
G. M. WEB $\mathrm{B}^{1} \dagger$, J. F. McKENZIE $\mathbf{E}^{1,3} \ddagger$ \\ A ND G. P. Z A N K $\mathbf{K}^{1,2}$ \\ ${ }^{1}$ Center for Space Plasma and Aeronomic Research, The University of Alabama in Huntsville, \\ Huntsville AL 35805, USA \\ ${ }^{2}$ Department of Space Science, The University of Alabama in Huntsville, Huntsville AL 35805, \\ USA \\ ${ }^{3}$ Department of Mathematics and Statistics, Durban University of Technology, \\ Steve Biko Campus, Durban South Africa, and School of Mathematical Sciences, University of \\ KwaZulu-Natal, Durban South Africa
}

(Received 13 November 2017)

A recent paper by Webb et al. (2014c) on multi-symplectic magnetohydrodynamics (MHD) using Clebsch variables in an Eulerian action principle with constraints is further extended. We relate a class of symplecticity conservation laws to a vorticity conservation law, and provide a corrected form of the Poincaré-Cartan differential form formulation of the system. We also correct some typographical errors (omissions) in Webb et al. (2014c). We show that the vorticity-symplecticity conservation law, that arises as a compatibility condition on the system, expressed in terms of the Clebsch variables is equivalent to taking the curl of the conservation form of the MHD momentum equation. We use the Cartan-Poincaré form to obtain a class of differential forms that represent the system using Cartan's geometric theory of partial differential equations.

\section{Introduction}

Multi-symplectic equations for Hamiltonian systems with two or more independent variables $x^{\alpha}$ have been developed as a useful extension of Hamiltonian systems with one evolution variable $t$. This development has connections with dual variational formulations of traveling wave problems (e.g. Bridges (1992)), and is useful in numerical schemes for Hamiltonian systems. Bridges and co-workers used the multi-symplectic approach to study linear and nonlinear wave propagation, generalizations of wave action, wave modulation theory, and wave stability problems (Bridqes (1997a), Bridqes (1997b)). Bridqes and Reich (2006) develop multi-symplectic difference schemes. Multisymplectic Hamiltonian systems have been studied by Marsden and Shkoller (1999) and Bridqes et al. (2005). Webb et al. (2007); Webb et al. (2008, 2014d) discuss traveling waves in multi-fluid plasmas using a multi-symplectic formulation. Holm et al. (1998) give an overview of Hamiltonian systems, semi-direct product Lie algebras and EulerPoincaré equations.

Cotter et al. (2007) developed a multi-symplectic, Euler-Poincaré formulation of fluid mechanics. They showed that multi-symplectic ideal fluid mechanics type systems are related to Clebsch variable formulations in which the Lagrange multipliers play the role of canonically conjugate momenta to the constrained variables. Thus, the Clebsch variable formulation involves a momentum map.

\section{$\dagger$ Email address for correspondence: gmw0002@uah.edu} $\ddagger$ deceased 
The main aim of the present paper is to correct and extend the analysis of the multisymplectic MHD equations derived by Webb et al. (2014c). Section 2 describes the MHD equations and the first law of thermodynamics. An overview of the key equations of the multi-symplectic MHD equations obtained by Webb et al. (2014c) is given in Section 3. We correct some typographical errors and present a more consistent description of the Cartan-Poincaré form for the system (section 4). The Cartan-Poincaré form is used to obtain a set of differential forms representing the system using Cartan's geometric theory of partial differential equations.

Section 5 concludes with a summary and discussion.

\section{The Model}

The magnetohydrodynamic equations are:

$$
\begin{aligned}
& \frac{\partial \rho}{\partial t}+\nabla \cdot(\rho \mathbf{u})=0, \\
& \frac{\partial}{\partial t}(\rho \mathbf{u})+\nabla \cdot\left[\rho \mathbf{u u}+\left(p+\frac{B^{2}}{2 \mu}\right) \mathbf{I}-\frac{\mathbf{B B}}{\mu}\right]=0, \\
& \frac{\partial S}{\partial t}+\mathbf{u} \cdot \nabla S=0, \\
& \frac{\partial \mathbf{B}}{\partial t}-\nabla \times(\mathbf{u} \times \mathbf{B})+\mathbf{u} \nabla \cdot \mathbf{B}=0
\end{aligned}
$$

where $\rho, \mathbf{u}, p, S$ and $\mathbf{B}$ are the gas density, fluid velocity, pressure, specific entropy, and magnetic induction $\mathbf{B}$ respectively, and $\mathbf{I}$ is the unit $3 \times 3$ dyadic. The gas pressure $p=$ $p(\rho, S)$ is a function of the density $\rho$ and entropy $S$, and $\mu$ is the magnetic permeability. Equations (2.1)-(2.2) are the mass and momentum conservation laws, (2.3) is the entropy advection equation and (2.4) is Faraday's equation in the MHD limit. In classical MHD, (2.1)-(2.4) are supplemented by Gauss' law:

$$
\nabla \cdot \mathbf{B}=0 .
$$

which implies the non-existence of magnetic monopoles. The MHD equations are closed by specifying an equation of state for the gas $U=U(\rho, S)$ where $U(\rho, S)$ is the internal energy per unit mass. The first law of thermodynamics has the form:

$$
T d S=d Q=d U+p d \tau \quad \text { where } \quad \tau=\frac{1}{\rho},
$$

where $\tau=1 / \rho$ is the specific volume. Using the internal energy per unit volume $\varepsilon=\rho U$ instead of $U,(2.6)$ may be written as:

$$
T d S=\frac{1}{\rho}(d \varepsilon-h d \rho) \quad \text { where } \quad h=\frac{\varepsilon+p}{\rho},
$$

is the enthalpy of the gas. Equation (2.7) gives the formulae:

$$
\rho T=\varepsilon_{S}, \quad h=\varepsilon_{\rho}, \quad p=\rho \varepsilon_{\rho}-\varepsilon,
$$

relating the temperature $T$, enthalpy $h$ and pressure $p$ to the internal energy density $\varepsilon(\rho, S)$. 


\section{Multi-Symplectic Appproach}

In this section we give a brief overview of the multi-symplectic formulation of MHD by Webb et al. (2014c).

\subsection{Clebsch Variable Action Principle}

Consider the MHD action (modified by constraints):

$$
J=\int d^{3} x d t L
$$

where

$$
\begin{aligned}
L= & \left\{\frac{1}{2} \rho u^{2}-\epsilon(\rho, S)-\frac{B^{2}}{2 \mu_{0}}\right\}+\phi\left(\frac{\partial \rho}{\partial t}+\nabla \cdot(\rho \mathbf{u})\right) \\
& +\beta\left(\frac{\partial S}{\partial t}+\mathbf{u} \cdot \nabla S\right)+\lambda\left(\frac{\partial \mu}{\partial t}+\mathbf{u} \cdot \nabla \mu\right) \\
& +\boldsymbol{\Gamma} \cdot\left(\frac{\partial \mathbf{B}}{\partial t}-\nabla \times(\mathbf{u} \times \mathbf{B})+\mathbf{u}(\nabla \cdot \mathbf{B})\right) .
\end{aligned}
$$

In (3.2) the Lagrange multipliers $\phi, \beta, \lambda$ and $\boldsymbol{\Gamma}$ ensure that the mass, entropy, Lin constraint and Faraday's equation are satisfied. Note we do not set $\nabla \cdot \mathbf{B}=0$ in our analysis. It can be set equal to zero after all variational calculations for the MHD system are finished. However, it does modify the Lagrange multiplier equation for $\boldsymbol{\Gamma}$ that ensures Faraday's equation is satisfied (see Morrison and Greene (1980), Morrison (1982). Morrison and Greene (1982), Holm and Kupershmidt (1983a) Holm and Kupershmidt (1983b) and Chandre et al. (2013) for further discussion of the MHD Poisson bracket). The Lagrangian in curly brackets equals the kinetic minus the potential energy (internal thermodynamic energy plus magnetic energy). The Lagrange multipliers $\phi, \beta, \lambda$, and $\boldsymbol{\Gamma}$ ensure that the mass, entropy, Lin constraint, Faraday equations are satisfied.

Stationary point conditions for the action are $\delta J=0 . \delta J / \delta \mathbf{u}=0$ gives the Clebsch representation for $\mathbf{M}=\rho \mathbf{u}$ :

$$
\mathbf{M}=\rho \mathbf{u}=\rho \nabla \phi-\beta \nabla S-\lambda \nabla \mu+\mathbf{B} \times(\nabla \times \mathbf{\Gamma})-\boldsymbol{\Gamma} \nabla \cdot \mathbf{B} .
$$

Setting $\delta J / \delta \phi, \delta J / \delta \beta, \delta J / \delta \lambda, \delta J / \delta \boldsymbol{\Gamma}$ equal to zero gives the mass, entropy advection, Lin constraint, and Faraday (magnetic flux conservation) constraint equations:

$$
\begin{aligned}
& \rho_{t}+\nabla \cdot(\rho \mathbf{u})=0, \\
& S_{t}+\mathbf{u} \cdot \nabla S=0, \\
& \mu_{t}+\mathbf{u} \cdot \nabla \mu=0, \\
& \mathbf{B}_{t}-\nabla \times(\mathbf{u} \times \mathbf{B})+\mathbf{u}(\nabla \cdot \mathbf{B})=0 .
\end{aligned}
$$

Similarly, setting $\delta J / \delta \rho, \delta J / \delta S, \delta J / \delta \mu, \delta J / \delta \mathbf{B}$ equal to zero gives evolution equations for the Clebsch potentials $\phi, \beta, \lambda$ and $\boldsymbol{\Gamma}$ as:

$$
\begin{aligned}
& -\left(\frac{\partial \phi}{\partial t}+\mathbf{u} \cdot \nabla \phi\right)+\frac{1}{2} u^{2}-h=0, \\
& \frac{\partial \beta}{\partial t}+\nabla \cdot(\beta \mathbf{u})+\rho T=0, \\
& \frac{\partial \lambda}{\partial t}+\nabla \cdot(\lambda \mathbf{u})=0, \\
& \frac{\partial \boldsymbol{\Gamma}}{\partial t}-\mathbf{u} \times(\nabla \times \boldsymbol{\Gamma})+\nabla(\boldsymbol{\Gamma} \cdot \mathbf{u})+\frac{\mathbf{B}}{\mu_{0}}=0 .
\end{aligned}
$$


Equation (3.5) is Bernoulli's equation. The $\nabla(\boldsymbol{\Gamma} \cdot \mathbf{u})$ term in (3.8) is associated with $\nabla \cdot \mathbf{B} \neq$ 0 .

\subsection{Multi-symplectic approach}

From Webb et al. (2014c), the MHD system was written in the multi-symplectic form:

$$
\mathrm{K}_{i j}^{\alpha} \frac{\partial z^{j}}{\partial x^{\alpha}}=\frac{\partial H}{\partial z^{i}},
$$

where the variables $z^{j}$ are defined as:

$$
\mathbf{z}=\left(u^{1}, u^{2}, u^{3}, \rho, S, \mu, B^{1}, B^{2}, B^{3}, \Gamma^{1}, \Gamma^{2}, \Gamma^{3}, \lambda, \beta, \phi\right)^{T} .
$$

The variables: $x^{\alpha}=(t, x, y, z)$ are the independent space and time variables, i.e. $x^{0}=t$, $x^{1}=x, x^{2}=y, x^{3}=z$. Below we use the notation $z_{, \alpha}^{s}=\partial z^{s} / \partial x^{\alpha}$. The constrained Lagrangian $L$ in (3.2) is written in the form:

$$
L=L_{0}+\sum_{s} L_{s}^{\alpha} z_{, \alpha}^{s}
$$

where

$$
L_{0}=\frac{1}{2} \rho u^{2}-\varepsilon(\rho, S)-\frac{B^{2}}{2 \mu}
$$

is the unconstrained Lagrangian.

The multi-symplectic Hamiltonian is given by the generalized Legendre transformation:

$$
H(z)=\sum_{s} L_{s}^{\alpha} z_{, \alpha}^{s}-L=-L_{0} \equiv-\left(\frac{1}{2} \rho u^{2}-\varepsilon(\rho, S)-\frac{B^{2}}{2 \mu}\right) .
$$

The fundamental one forms $\omega^{\alpha}(\alpha=0,1,2,3)$ of the multi-symplectic system (3.9) are defined as:

$$
\omega^{\alpha}=L_{j}^{\alpha}(z) d z^{j}
$$

Taking the exterior derivative of the $\omega^{\alpha}$ gives the formulae

$$
\kappa^{\alpha}=d \omega^{\alpha}=\frac{1}{2} \mathrm{~K}_{i j}^{\alpha} d z^{i} \wedge d z^{j},
$$

which, in turn, gives the formulae:

$$
\mathrm{K}_{i j}^{\alpha}=\left(\frac{\partial L_{j}^{\alpha}}{\partial z^{i}}-\frac{\partial L_{i}^{\alpha}}{\partial z^{j}}\right),
$$

for the skew symmetric matrices in (3.9).

For the variables $z^{j}$ in (3.10) the one forms $\omega^{\alpha}$ are given by:

$$
\begin{aligned}
\omega^{0} & =\phi d \rho+\beta d S+\lambda d \mu+\boldsymbol{\Gamma} \cdot d \mathbf{B}, \\
\omega^{i} & =u^{i}(\beta d S+\lambda d \mu+\phi d \rho)+\rho \phi d u^{i}+\boldsymbol{\Gamma} \cdot \mathbf{B} d u^{i}-B^{i}(\boldsymbol{\Gamma} \cdot d \mathbf{u})+u^{i}(\boldsymbol{\Gamma} \cdot d \mathbf{B}),
\end{aligned}
$$

where $1 \leqslant i \leqslant 3$.

The pullback conservation laws:

$$
G_{\beta}=D_{\alpha}\left(L_{j}^{\alpha}(z) z_{, \beta}^{j}-L \delta_{\beta}^{\alpha}\right)=0,
$$


follow from the pullback of the identities:

$$
\begin{aligned}
& \left(L_{j}^{\alpha} d z^{j}\right)_{, \alpha}=d\left\{L_{j}^{\alpha}(z) z_{, \alpha}^{j}-H(z)\right\}=d L, \\
& \left(L_{j}^{\alpha} d z^{j}\right)_{, \alpha}=\left(L_{j}^{\alpha} z_{, \beta}^{j} d x^{\beta}\right)_{, \alpha}=\left(L_{j}^{\alpha} z_{, \beta}^{j}\right)_{, \alpha} d x^{\beta}=d L=\frac{\partial L^{\alpha}}{\partial x^{\beta}} d x^{\beta} .
\end{aligned}
$$

(see proposition 4.1 of Webb et al. (2014c) for more detail).

The pullback equation $\kappa_{, \alpha}^{\alpha}=0$ where $\kappa^{\alpha}=d \omega^{\alpha}$ gives rise to the symplecticity or phase-space conservation laws (structural conservation laws):

$$
D_{\alpha}\left(\mathrm{K}_{i j}^{\alpha} z_{, \beta}^{i} z_{, \gamma}^{j}\right)=0, \quad \beta<\gamma .
$$

These conservation laws can also be written in the form:

$$
D_{\beta} G_{\gamma}-D_{\gamma} G_{\beta}=D_{\alpha}\left(\mathrm{K}_{i j}^{\alpha} z_{, \beta}^{i} z_{, \gamma}^{j}\right)=0,
$$

i.e., the symplecticity conservation laws (3.21) are compatibility conditions for the pullback conservation laws (3.18).

The pullback conservation law (3.18) for $\beta=0$ (Webb et al. (2014c), equation (5.39)) reduces to the energy conservation law:

$$
G_{0}=-\left\{\frac{\partial}{\partial t}\left(\frac{1}{2} \rho u^{2}+\varepsilon(\rho, S)+\frac{B^{2}}{2 \mu}\right)+\nabla \cdot\left(\rho \mathbf{u}\left(\frac{1}{2} u^{2}+h\right)+\frac{\mathbf{E} \times \mathbf{B}}{\mu}\right)\right\}=0,
$$

where $h=(\varepsilon+p) / \rho$ is the enthalpy of the gas, $\mathbf{E}=-\mathbf{u} \times \mathbf{B}$ is the electric field, and $\mathbf{E} \times \mathbf{B} / \mu$ is the Poynting flux. Similarly, the pullback conservation laws (3.18) for $\beta=i$ $(1 \leqslant i \leqslant 3)$ give rise to the MHD momentum conservation equation:

$$
G^{i}=-\left\{\frac{\partial}{\partial t}(\rho \mathbf{u})+\nabla \cdot\left[\rho \mathbf{u} \otimes \mathbf{u}+\left(p+\frac{B^{2}}{2 \mu}\right) \mathbf{I}-\frac{\mathbf{B} \otimes \mathbf{B}}{\mu}\right]\right\}^{i}=0
$$

(Webb et al. (2014c), equation (5.41)).

\section{Extensions, comments and corrections}

The symplecticity conservation laws (3.20)- (3.21) have a generalized curl form. Consider the symplecticity laws (3.21) for $1 \leqslant i, k \leqslant 3$, namely:

$$
\Omega_{i k}=D_{i} G_{k}-D_{k} G_{i}=0 .
$$

Introduce the dual of the tensor $\Omega_{i k}$ defined as:

$$
V^{p}=-\frac{1}{2} \varepsilon_{p i k} \Omega_{i k}=-(\nabla \times \mathbf{G})^{p},
$$

where $\nabla \times \mathbf{G}$ is the spatial curl of $\mathbf{G}$. Taking into account the momentum conservation law (3.23) for $\mathbf{G}$, (4.2) reduces to:

$$
\nabla \times \mathbf{G}=-\left\{\frac{\partial}{\partial t} \nabla \times \mathbf{M}+\nabla \times\left[\nabla \cdot\left(\mathbf{M} \otimes \mathbf{u}-\frac{\mathbf{B} \otimes \mathbf{B}}{\mu}\right)\right]\right\}=0,
$$

where

$$
\mathbf{M}=\rho \mathbf{u},
$$

is the momentum density or mass flux $\mathbf{M}$ of the MHD fluid. Note there is no contribution from the magnetic pressure $\left(B^{2} /(2 \mu)\right)$ and gas pressure $(p)$ gradient force terms in (4.3) because $\nabla \times \nabla\left(p+B^{2} /(2 \mu)\right)=0$ when one takes the curl of the momentum equation 
(3.23). The evolution of $\nabla \times \mathbf{M}$ in (4.3) is thus determined by the inertia and magnetic tension components:

$$
\mathbf{M} \otimes \mathbf{u}-\frac{\mathbf{B} \otimes \mathbf{B}}{\mu},
$$

of the MHD stress-energy tensor. This suggests that (4.3) describes Alfvénic type disturbances, in which both fluid spin and magnetic tension forces are part of the dynamics. Equation (4.3) can also be expressed in the conservation law form:

$$
\frac{\partial}{\partial t} \nabla \times \mathbf{M}+\nabla_{s}\left[\nabla \times\left(\mathbf{M} u^{s}-\frac{\mathbf{B} B^{s}}{\mu}\right)\right]=0 .
$$

Pressure gradient forces play no role in the vorticity-like conservation laws (4.3) and (4.6).

The symplecticity conservation law (4.3) is different than that obtained by taking the curl of Euler momentum equation in the form $d \mathbf{u} / d t=\mathbf{F}$ where $\mathbf{F}$ is the net force on the fluid element, to obtain an equation for the evolution of the fluid vorticity $\boldsymbol{\omega}=$ $\nabla \times \mathbf{u}$. Webb et al. (2014a) Webb et al. (2014b) and Webb and Anco (2015) obtained a conservation law in ideal fluid mechanics (i.e. for $\mathbf{B}=0$ ) for the generalized vorticity

$$
\boldsymbol{\Omega}=\boldsymbol{\omega}+\nabla r \times \nabla S \quad \text { where } \quad \boldsymbol{\omega}=\nabla \times \mathbf{u},
$$

is the fluid vorticity and $r$ satisfies the equation:

$$
\frac{d r}{d t}=\left(\frac{\partial}{\partial t}+\mathbf{u} \cdot \nabla\right) r=-T
$$

Here $r=\beta / \rho$ where $\beta$ is the Clebsch potential that ensures $d S / d t=0$ in the Eulerian, Clebsch variational approach (e.g. Zakharov and Kuznetson (1997)) and $d / d t=\partial / \partial t+$ $\mathbf{u} \cdot \nabla$ is the Lagrangian time derivative following the flow. Webb et al. (2014a). Webb et al. (2014b) and Webb and Anco (2015) show that for fluid dynamics $(\mathbf{B}=0)$ the modified vorticity flux $\boldsymbol{\Omega} \cdot d S$ is advected or Lie dragged with the flow, i.e.,

$$
\frac{d}{d t}(\boldsymbol{\Omega} \cdot d S)=\left[\frac{\partial \boldsymbol{\Omega}}{\partial t}-\nabla \times(\mathbf{u} \times \boldsymbol{\Omega})+\mathbf{u}(\nabla \cdot \boldsymbol{\Omega})\right] \cdot d S=0 .
$$

The conservation law (4.9) and the associated conservation law for the modified fluid helicity $\mathbf{u} \cdot \boldsymbol{\Omega}$ are nonlocal conservation laws that depend on the nonlocal variable $r=$ $-\int_{0}^{t} T(\mathbf{x}, t) d t$ where the integration is with respect to the Lagrangian time $t$ (e.g. Webb et al. (2014a); Webb et al. (2014b)). Conservation law (4.9) in fluid dynamics is analogous to Faraday's equation in MHD. Note that $\nabla \cdot \boldsymbol{\Omega}=0$ in (4.9).

There is another symplecticity conservation law obtained from (3.21) for the case $\beta=0$ and $\gamma=1,2,3$. In that case (3.21) reduces to:

$$
\frac{\partial}{\partial t} \mathbf{G}-\nabla G_{0}=0
$$

where $\mathbf{G}=0$ is the momentum equation (3.23) and $G_{0}=0$ is the energy conservation equation (3.22).

The general form of the symplecticity equations for MHD using Eulerian Clebsch potentials were given in Webb et al. (2014c) [equations (5.44) et seq. of that paper]. There were some typographical errors in the flux $F_{a b}^{k}$, indicated below. The general symplecticity conservation laws obtained by Webb et al. (2014c) have the form:

$$
\frac{\partial}{\partial t}\left(F_{a b}^{0}\right)+\frac{\partial}{\partial x^{k}}\left(F_{a b}^{k}\right)=0
$$


where

$$
F_{a b}^{0}=\frac{\partial(\phi, \rho)}{\partial\left(x^{a}, x^{b}\right)}+\frac{\partial(\beta, S)}{\partial\left(x^{a}, x^{b}\right)}+\frac{\partial(\lambda, \mu)}{\partial\left(x^{a}, x^{b}\right)}+\frac{\partial\left(\Gamma_{s}, B^{s}\right)}{\partial\left(x^{a}, x^{b}\right)}
$$

and

$$
\begin{aligned}
F_{a b}^{k}= & -\frac{\partial\left(\rho u^{k}, \phi\right)}{\partial\left(x^{a}, x^{b}\right)}+\frac{\partial\left(\beta u^{k}, S\right)}{\partial\left(x^{a}, x^{b}\right)}+\frac{\partial\left(\lambda u^{k}, \mu\right)}{\partial\left(x^{a}, x^{b}\right)} \\
& +\frac{\partial\left(\Gamma_{s} B^{s}, u^{k}\right)}{\partial\left(x^{a}, x^{b}\right)}+\frac{\partial\left(\Gamma_{s} u^{k}, B^{s}\right)}{\partial\left(x^{a}, x^{b}\right)}-\frac{\partial\left(\Gamma_{s} B^{k}, u^{s}\right)}{\partial\left(x^{a}, x^{b}\right)} .
\end{aligned}
$$

In (4.13) $1 \leqslant k \leqslant 3$. The fourth term on the right handside of (4.13) was missed in equation (5.48) in Webb et al. (2014c). Also in (4.13) we used the identity:

$$
\frac{\partial\left(\phi u^{k}, \rho\right)}{\partial\left(x^{a}, x^{b}\right)}+\frac{\partial\left(\rho \phi, u^{k}\right)}{\partial\left(x^{a}, x^{b}\right)}=-\frac{\partial\left(\rho u^{k}, \phi\right)}{\partial\left(x^{a}, x^{b}\right)},
$$

to simplify (5.48) of Webb et al. (2014c). The derivation of the symplecticity conservation laws (4.3) and (4.10) using the general symplecticity laws (4.11) and using (3.3)-(3.8) is a non-trivial algebraic exercise.

\subsection{Differential forms approach}

Proposition 4.3 in Webb et al. (2014c) contains flaws. A consistent approach to the multisymplectic equations using differential forms for 1D Lagrangian gas dynamics was given by Webb (2015). Webb and Anco (2015) have given the corresponding theory for multidimensional, ideal, compressible, Lagrangian gas dynamics. Below we use differential forms to describe the Eulerian, Clebsch variable MHD variational principle of Webb et al. (2014c).

Proposition 4.1. The multi-symplectic system (3.9) is a stationary point of the action:

$$
J=\int \psi^{*}(\Theta)=\int L d V,
$$

where $\psi^{*}(\Theta)$ is the pullback of the differential form $\Theta$ given below, namely:

$$
\begin{aligned}
\Theta & =\omega^{\alpha} \wedge d \tilde{x}^{\alpha}-H d V, \quad \omega^{\alpha}=L_{j}^{\alpha} d z^{j}, \\
d V & \left.=d t \wedge d x \wedge d y \wedge d z, \quad d \tilde{x}^{\alpha}=\partial_{\alpha}\right\lrcorner d V \equiv(-1)^{\alpha} d x_{0} \wedge \ldots d x^{\alpha-1} \wedge d x^{\alpha+1} \ldots \wedge d x^{n},
\end{aligned}
$$

where we use the notation $\left(x^{0}, x^{1}, x^{2}, x^{3}\right)=(t, x, y, z)$, and $L$ is the constrained Lagrangian (3.2).

Proof. The pullback of the form $\Theta$ is given by:

$$
\begin{aligned}
\psi^{*}(\Theta) & =\psi^{*}\left(L_{j}^{\alpha} d z^{j} \wedge d \tilde{x}_{\alpha}-H d V\right) \\
& =L_{j}^{\alpha} \frac{\partial z^{j}}{\partial x^{s}} d x^{s} \wedge d \tilde{x}^{\alpha}-H d V .
\end{aligned}
$$

However,

$$
d x^{s} \wedge d \tilde{x}_{\alpha}=d x^{s} \wedge(-1)^{\alpha} d x^{0} \ldots \wedge d x^{\alpha-1} \wedge d x^{\alpha+1} \ldots \wedge d x^{n} \equiv(-1)^{2 \alpha} \delta_{\alpha}^{s} d V .
$$

Thus,

$$
\psi^{*}(\Theta)=\left(L_{j}^{\alpha} \frac{\partial z^{j}}{\partial x^{\alpha}}-H\right) d V \equiv L d V
$$

where $L$ is the multi-symplectic Lagrangian (3.10). 
The stationary point conditions, $\delta J / \delta z^{i}=0$, give the Euler-Lagrange equations:

$$
\frac{\delta J}{\delta z^{i}}=\frac{\partial L}{\partial z^{i}}-\frac{\partial}{\partial x^{j}}\left(\frac{\partial L}{\partial z_{, j}^{i}}\right)=\mathrm{K}_{i j}^{\alpha} \frac{\partial z^{j}}{\partial x^{\alpha}}-\frac{\partial H}{\partial z^{i}}=0,
$$

which is the multi-symplectic system (3.9) (see also Hydon (2005)).

Proposition 4.2. Consider the variational functional:

$$
G[\Omega]=\int_{M} \Omega
$$

where

$$
\Omega=d \Theta=d \omega^{\alpha} \wedge d \tilde{x}_{\alpha}-d H \wedge d V
$$

and $M$ is a region of the jet space (fiber bundle space) with boundary $\partial M$, in which the $z^{s}$ are taken as independent of the base variables $x^{\alpha}(\alpha=0,1,2,3)$. Consider the variational principle:

$$
\delta G[\Omega]=\int_{M} \mathcal{L}_{\mathbf{V}}(\Omega)=0
$$

where

$$
\mathcal{L}_{\mathbf{V}}=\frac{d}{d \epsilon}=V^{i} \frac{\partial}{\partial z^{i}}
$$

is the Lie derivative with respect to the arbitrary, but smooth vector field $\mathbf{V}$. The variational equation $\delta G[\Omega]=0$ reduces to:

$$
\delta G[\Omega]=\int_{\partial M} V^{p} \beta_{p}=0,
$$

where the forms $\beta_{p}$ are given by the formulae:

$$
\left.\beta_{p}=\frac{\partial}{\partial z^{p}}\right\lrcorner \Omega=\mathrm{K}_{p j}^{\alpha} d z^{j} \wedge d \tilde{x}_{\alpha}-\frac{\partial H}{\partial z^{p}} d V .
$$

$(1 \leqslant p \leqslant N)$. Because the $V^{p}$ are arbitrary smooth functions of the $z^{s}$, the variational principle $\delta G[\Omega]=0$ implies:

$$
\beta_{p}=0, \quad 1 \leqslant p \leqslant N
$$

The pullback of the forms $\left\{\beta_{p}\right\}$ to the base manifold gives the equations:

$$
\tilde{\beta}_{p}=\left(\mathrm{K}_{p j}^{\alpha} \frac{\partial z^{j}}{\partial x^{\alpha}}-\frac{\partial H}{\partial z^{p}}\right) d V=0 .
$$

Thus, the sectioned forms $\tilde{\beta}_{p}$ vanish on the solution manifold of the multi-symplectic system (3.9), and the $\left\{\beta_{p}\right\}$ can be used as a basis of Cartan forms describing the system (3.9).

Proof.The proof is essentially the same as that given by Webb (2015) for the case of 1D gas dynamics. A critical component of the proof is the use of Cartan's magic formula:

$$
\left.\left.\left.\mathcal{L}_{\mathbf{V}}(\Omega)=\mathbf{V}\right\lrcorner d \Omega+d(\mathbf{V}\lrcorner \Omega\right)=d(\mathbf{V}\lrcorner \Omega\right),
$$

where we used the facts $\Omega=d \Theta$ and $d \Omega=d d \Theta=0$. Using (4.29) and Stokes theorem, (4.23) reduces to:

$$
\left.\left.\left.\delta G[\Omega]=\int_{M} d(\mathbf{V}\lrcorner \Omega\right)=\int_{\partial M} \mathbf{V}\right\lrcorner \Omega=\int_{\partial M} V^{p}\left(\frac{\partial}{\partial z^{p}}\right\lrcorner \Omega\right)=\int_{\partial M} V^{p} \beta_{p}=0,
$$


which verifies (4.25). The formula (4.26) for $\beta_{p}$ is obtained by using (3.15) for $d \omega^{\alpha}$ and (4.22) for $\Omega$, to obtain:

$$
\left.\left.\beta_{p}=\frac{\partial}{\partial z^{p}}\right\lrcorner \Omega=\frac{\partial}{\partial z^{p}}\right\lrcorner\left(\frac{1}{2} K_{i j}^{\alpha} d z^{i} \wedge d z^{j} \wedge d \tilde{x}_{\alpha}-\frac{\partial H}{\partial z^{a}} d z^{a} \wedge d V\right),
$$

Using the skew symmetry of $\mathrm{K}_{i j}^{\alpha}$ and $d z^{i} \wedge d z^{j}$, (4.31) reduces to the expression (4.26) for $\beta_{p}$. This completes the proof.

\subsection{The differential forms $\beta_{p}$}

The differential forms $\left.\beta_{p}=\partial_{z^{p}}\right\lrcorner \Omega$ in (4.26) may be used to represent the MHD system described by the Clebsch variable variational principle. The dependent variables $\mathbf{z}$ are listed in (3.10). In the time evolution variational principle (e.g. Zakharov and Kuznetsov (1997), the fluid velocity $\mathbf{u}$ is expressed in terms of the Clebsch potentials, and is eliminated from the Hamiltonian density $H=(1 / 2) \rho u^{2}+\varepsilon(\rho, S)+B^{2} /(2 \mu)$ and $(\rho, \phi),(S, \beta)$, $(\mu, \lambda),(\mathbf{B}, \boldsymbol{\Gamma})$ are canonically conjugate pairs in the canonical Poisson bracket. We use the notation:

$$
\left.\beta^{z^{i}}=\partial_{z^{i}}\right\lrcorner \Omega
$$

where the Cartan Poincaré form $\Omega$ in (4.22) has the form:

$$
\Omega=d \omega^{0} \wedge d \tilde{x}_{0}+d \omega^{k} \wedge d \tilde{x}_{k}-\frac{\partial H}{\partial z^{p}} d z^{p} \wedge d V
$$

where the differential forms $\omega^{0}$ and $\omega^{k}$ are listed in (3.17). From (4.32) and (4.33)

$$
\left.\beta^{z^{i}}=\partial_{z^{i}}\right\lrcorner\left\{d \omega^{0} \wedge d \tilde{x}_{0}+d \omega^{k} \wedge d \tilde{x}_{k}-\frac{\partial H}{\partial z^{p}} d z^{p} \wedge d V\right\} .
$$

Using (3.17) and (4.34) we obtain:

$$
\beta^{u^{i}}=\left(\beta d S+\lambda d \mu-\rho d \phi-B^{s} d \Gamma_{s}\right) \wedge d \tilde{x}_{i}+\left(\Gamma_{i} d B^{k}+B^{k} d \Gamma_{i}\right) \wedge d \tilde{x}_{k}+\rho u^{i} d V,
$$

for the differential forms associated with $\mathbf{u}$. Using the identity

$$
d x^{a} \wedge d \tilde{x}_{i}=\delta_{i}^{a} d V
$$

the sectioned form equation $\tilde{\beta}^{u^{i}}=0$ yields the expression:

$$
\rho \mathbf{u}=\rho \nabla \phi-\beta \nabla S-\lambda \nabla \mu+\mathbf{B} \cdot(\nabla \boldsymbol{\Gamma})^{T}-\mathbf{B} \cdot \nabla \boldsymbol{\Gamma}-\boldsymbol{\Gamma} \nabla \cdot \mathbf{B},
$$

which is equivalent to the Clebsch expansion for the mass flux $\rho \mathbf{u}$ given in (3.3).

The differential form $\beta^{\rho}$ is given by:

$$
\left.\left.\left.\beta^{\rho}=\partial_{\rho}\right\lrcorner \Omega=\left(\partial_{\rho}\right\lrcorner d \omega^{0}\right) \wedge d \tilde{x}_{0}+\left(\partial_{\rho}\right\lrcorner d \omega^{k}\right) \wedge d \tilde{x}^{k}-\frac{\partial H}{\partial \rho} d V .
$$

Using (3.14) for $H$, we obtain:

$$
\frac{\partial H}{\partial \rho}=-\left(\frac{1}{2} u^{2}-\varepsilon_{\rho}\right)=-\left(\frac{1}{2} u^{2}-h\right),
$$

where $h=(\varepsilon+p) / \rho$ is the gas enthalpy. Substituting (4.39) in (4.38) gives

$$
\begin{aligned}
\beta^{\rho} & =-\left(d \phi \wedge d \tilde{x}_{0}+u^{k} d \phi \wedge d \tilde{x}_{k}\right)+\left(\frac{1}{2} u^{2}-h\right) d V, \\
\tilde{\beta}^{\rho} & =-\left[\frac{d \phi}{d t}-\left(\frac{1}{2} u^{2}-h\right)\right] d V,
\end{aligned}
$$


for the differential form $\beta^{\rho}$ and for the sectioned form $\tilde{\beta}^{\rho}$. Note that $\tilde{\beta}^{\rho}=0$ is equivalent to Bernoulli's equation (3.5). Similarly, we obtain:

$$
\begin{aligned}
& \left.\beta^{S}=\partial_{S}\right\lrcorner \Omega=-d \beta \wedge d \tilde{x}_{0}-d\left(\beta u^{k}\right) \wedge d \tilde{x}_{k}-\rho T d V, \\
& \tilde{\beta}^{S}=-\left(\frac{\partial \beta}{\partial t}+\nabla \cdot(\beta \mathbf{u})+\rho T\right) d V .
\end{aligned}
$$

The equation $\tilde{\beta}^{S}=0$ corresponds to $(3.6)$ for $\beta$.

Following the above procedure we obtain the equations:

$$
\begin{aligned}
\beta^{\mu} & \left.=\partial_{\mu}\right\lrcorner \Omega=-\left[d \lambda \wedge d \tilde{x}_{0}+d\left(\lambda u^{k}\right) \wedge d \tilde{x}_{k}\right], \\
\beta^{B^{i}} & =-\left[d \Gamma_{i} \wedge d \tilde{x}_{0}+u^{k} d \Gamma_{i} \wedge d \tilde{x}_{k}+\Gamma_{s} d u^{s} \wedge d \tilde{x}_{i}+\frac{B^{i}}{\mu} d V\right], \\
\beta^{\Gamma_{i}} & =d B^{i} \wedge d \tilde{x}_{0}+\left(B^{i} d u^{k}+u^{k} d B^{i}-B^{k} d u^{i}\right) \wedge d \tilde{x}_{k}, \\
\beta^{\lambda} & \left.=\partial_{\lambda}\right\lrcorner \Omega=\left(d \mu \wedge d \tilde{x}_{0}+u^{k} d \mu \wedge d \tilde{x}_{k}\right), \\
\beta^{\beta} & \left.=\partial_{\beta}\right\lrcorner \Omega=d S \wedge d \tilde{x}_{0}+u^{k} d S \wedge d \tilde{x}_{k}, \\
\beta^{\phi} & \left.=\partial_{\phi}\right\lrcorner \Omega=d \rho \wedge d \tilde{x}_{0}+\left(u^{k} d \rho+\rho d u^{k}\right) \wedge d \tilde{x}_{k} .
\end{aligned}
$$

The pullback of the above equations, i.e. $\tilde{\beta}^{z^{p}}=0$, gives the evolution equations for $(\lambda, \boldsymbol{\Gamma}, \mathbf{B}, \mu, S, \rho)$ listed in (3.4) $-(3.8)$. The differential form equations $\beta^{z^{p}}=0$ thus represent the partial differential equation system (3.9).

It is not obvious that the system of forms $\left\{\beta^{z^{p}}\right\}$ above is a closed ideal. A check on the closure of the forms for the case of non-barotropic, 1D gas dynamics (i.e. $\mathbf{B}=0$ ) indicates that the ideal of forms $\mathcal{I}=\left\{\beta^{u}, \beta^{\rho}, \beta^{S}, \beta^{\beta}, \beta^{\phi}\right\}$ can be closed by adjoining the form $d \beta^{u}$. The ideal $\mathcal{I}$ is closed for the case of a barotropic gas. The Cartan approach to Lie symmetries requires that $\mathcal{I}$ is a closed ideal (e.g. Harrison and Estabrook (1971)). The ideal is closed if $d \beta_{i}=c_{i j} \wedge \beta^{z^{j}}$, where the $c_{i j}$ are forms. It should be noted that the ideal of forms obtained by Webb (2015) for 1D, Lagrangian, multi-symplectic gas dynamics is closed. The ideal of forms for multi-dimensional, Lagrangian. compressible gas dynamics obtained from the Cartan-Poincaré form is also a closed ideal (Webb and Anco (2015)). This suggests that the ideal of forms using the Clebsch variable description has a more complicated structure than the set of forms that arise in the Lagrangian variational approach. In general, this question requires more work and lies beyond the scope of the present paper.

\section{Summary and Concluding Remarks}

In this paper, we have corrected some flaws in the paper by Webb et al. (2014c) on multi-symplectic MHD. This includes the correction of some typographic errors in the expressions for the conserved fluxes in the symplecticity conservation laws written in terms of the Clebsch potentials in (4.11)-(4.13) and in particular expression (4.13) for $F_{a b}^{k}$ has been corrected. We pointed out that the vorticity-type symplecticity conservation law is equivalent to taking the curl of the MHD momentum equation in the form (4.6). This conservation law has conserved density $\nabla \times \mathbf{M}$ where $\mathbf{M}=\rho \mathbf{u}$ is the momentum density of the MHD fluid. Pressure gradient forces due to the combined gas and magnetic pressures (i.e. $\nabla\left(p+p_{B}\right)$, where $p_{B}=B^{2} /(2 \mu)$ is the magnetic pressure and $p$ is the gas pressure), play no role in this conservation law. The conservation law (4.6) describes fluid motions with spin and rotation (e.g; as in an Alfvén wave). This symplecticity conservation law is different than that obtained by Webb and Anco (2015) in ideal 
Lagrangian fluid mechanics. In the latter paper the vorticity symplecticity conservation law involved $\boldsymbol{\Omega}=\boldsymbol{\omega}+\nabla r \times \nabla S$ where $\boldsymbol{\omega}=\nabla \times \mathbf{u}$ is the fluid vorticity and $r=\beta / \rho$ where $\beta$ is the Clebsch potential that enforces entropy conservation following the flow. This is a nonlocal conservation law as it involves the nonlocal variable $r$. It has the same form as Faraday's equation in MHD except that $\mathbf{B}$ is replaced by $\boldsymbol{\Omega}$. A further symplecticity conservation law is due to a compatibility condition which requires that time derivative of the the momentum conservation equation minus the gradient of the energy conservation equation is zero. We provided a more consistent treatment of variational principles associated with the Cartan-Poincaré form than that given in Webb et al. (2014c), and its relation to Cartan's geometric theory of partial differential equations using differential forms (Sections 4.1 and 4.2). Cendra and Capriote (2013) suggest that the formulation of multi-symplectic systems using exterior differential forms, is a natural approach in taking into account integrability conditions in the theory.

\section{Acknowledgements}

GMW acknowledges discussions with Darryl Holm and Phil. Morrison on multi-symplectic MHD. GMW is supported in part by NASA grant NNX15A165G. GPZ was supported in part by NASA grants NN05GG83G and NSF grant nos. ATM-03-17509 and ATM-0428880 .

\section{REFERENCES}

Bridges, T. J. 1992, Spatial Hamiltonian structure, energy flux and the water-wave problem. Proc. Roy. Soc. London,439, 297-315.

Bridges, T.J., 1997a, Multi-symplectic structures and wave propagation, Math. Proc. Camb. Philos. Soc., 121, 147-190.

Bridges, T. J., 1997b, A geometric formulation of the conservation of wave action and its implications for signature and classification of instabilities, Proc. Roy. Soc. A, 453, 1365-1395 (1997b).

Bridges, T. J. Hydon, P. E. and Reich, S. 2005, Vorticity and symplecticity in Lagrangian fluid dynamics, J. Phys. A: Math. Gen. 38 1403-1418.

Bridges, T. J. and Reich, S. 2006, Numerical methods for Hamiltonian PDEs, J. Phys. A, Math. Gen., 39, 5287-5320.

Bridges, T. J. 2006, Canonical multi-symplectic structure on the total exterior algebra bundle, Proc. Roy. Soc. London, A, 462, 1531-1551.

Bridges, T. J., Hydon, P. E. and Lawson, J.K. 2010, Multi-symplectic structures and the variational bi-complex, Math. Proc. Cambridge Phil. Soc., issue 1, (Jan. 2010), pp 159-178.

Chandre, C., de Guillebon, L., Back, A., Tassi, E. and Morrison, P. J. 2013, On the use of projectors for Hamiltonian systems and their relationship with Dirac brackets, J. Phys. A, Math. and theoret., 46, 125203 (14pp), doi:10.10.1088/1751-8133/46/12/125203.

Cendra, H, and Caprioti, S. 2013, Cartan algorithm and Dirac constraints for Griffiths variational problems, available at http://arxiv.org/abs/1309.4080v1

Cotter, C. J., Holm, D. D., and Hydon, P. E., 2007, Multi-symplectic formulation of fluid dynamics using the inverse map, Proc. Roy. Soc. Lond. A, 463, 2617-2687 (2007).

Holm, D. D. and Kupershmidt, B. A. 1983a, Poisson brackets and Clebsch representations for magnetohydrodynamics, multi-fluid plasmas and elasticity, Physica D, 6D, 347-363.

Holm, D. D. and Kupershmidt, B. A. 1983b, noncanonical Hamiltonian formulation of ideal magnetohydrodynamics, Physica D, 7D, 330-333.

Holm, D.D., Marsden, J.E. and Ratiu, T.S. 1998, The Euler-Lagrange equations and semiproducts with application to continuum theories, Adv. Math., 137, 1-81.

Hydon, P. E., 2005, Multi-symplectic conservation laws for differential and differential-difference equations, Proc. Roy. Soc. A, 461, 1627-1637 (2005). 
Marsden, J. E. and Shkoller, S. 1999, Multi-symplectic geometry, covariant Hamiltonians and Water Waves, Math. Proc. Camb. Phil. Soc., 125, 553-575.

Morrison, P. J. 1982, Poisson brackets for fluids and plasmas, in Mathematical Methods in Hydrodynamics and Integrability of dynamical Systems, AIP Proc. Conf., 88, ed M. Tabor and Y. M. Treve, pp 13-46.

Morrison, P.J. and Greene, J.M. 1980, Noncanonical Hamiltonian density formulation of hydrodynamics and ideal magnetohydrodynamics, Phys. Rev. Lett., 45, 790-794.

Morrison, P.J. and Greene, J.M. 1982, Noncanonical Hamiltonian density formulation of hydrodynamics and ideal magnetohydrodynamics, (Errata), Phys. Rev. Lett., 48, 569.

Webb, G. M. 2015, Multi-symplectic, Lagrangian, one-dimensional gas dynamics, J. Math. Phys., 56, 053101 (20pp.), also available at http://arxiv.org/abs/1408.4028v4

Webb, G. M.; McKenzie, J. F.; Mace, R. L.; Ko, C. M.; Zank, G. P. 2007, Dual variational principles for nonlinear traveling waves in multifluid plasmas, Phys. of Plasmas, 4, Issue 8, pp. 082318-082318-17, doi:10.1063/1.2757154

Webb, G. M., Ko, C. M., Mace, R.L., McKenzie, J.F. and Zank, G.P. 2008, Integrable, oblique travelling waves in charge neutral, two-fluid plasmas, Nonl. Proc. Geophys., 15, 179-208.

Webb, G. M., Dasgupta, B., McKenzie, J. F., Hu, Q., and Zank, G.P. 2014a: Local and nonlocal advected invariants and helicities in magnetohydrodynamics and gas dynamics I: Lie dragging approach, J. Phys. A., Math. and Theoret., 47, (2014) 095501 (33pp). doi:10.1088/1751-8113/49/9/095501, preprint available at http://arxiv.org/abs/1307.1105.

Webb, G. M., Dasgupta, B., McKenzie, J. F., Hu, Q., and Zank, G.P. 2014b: Local and nonlocal advected invariants and helicities in magnetohydrodynamics and gas dynamics II: Noether's theorems and Casimirs, J. Phys. A., Math. and Theoret., 47 (2014) 095502 (31pp), doi:10.1088/1751-8113/47/9/095502, preprint available at http://arxiv.org/abs/1307.1038.

Webb, G. M., McKenzie, J.F. and Zank, G.P. 2014c, Multisymplectic magnetohydrodynamics, J. Plasma Phys., 80, part 5, pp. 707-743, doi:10.1017/S0022377814000257, also available at http://arxiv.org/abs/1312.4890v4

Webb, G. M., Burrows, R. H., Ao, X., and Zank, G.P. 2014d, Ion acoustic travelling waves, J. Plasma Phys., 80, part 2, pp. 147-171, doi:10.1017/S0022377813001013, preprint at http://arxiv.org/abs/1312.6406

Webb, G. M. and Anco, S.C. 2015, Vorticity and Symplecticity in Multi-Symplectic, Lagrangian Gas Dynamics, J. Phys. A, Math. and Theoret., submitted May 6, 2015.

Zakharov, V. E. and Kuznetsov, E.A. 1997, Hamiltonian formalism for nonlinear waves, PhysicsUspekhi, 40, (11), 1087-1116. 